\title{
Pell Graphs
}

\author{
Emanuele Munarini
}

Politecnico di Milano, Dipartimento di Matematica, P.zza Leonardo da Vinci 32, 20133 Milano, Italy.

\begin{abstract}
In this paper, we introduce the Pell graphs, a new family of graphs similar to the Fibonacci cubes. They are defined on certain ternary strings (Pell strings) and turn out to be subgraphs of Fibonacci cubes of odd index. Moreover, as well as ordinary hypercubes and Fibonacci cubes, Pell graphs have several interesting structural and enumerative properties. Here, we determine some of them. Specifically, we obtain a canonical decomposition giving a recursive structure, some basic properties (bipartiteness and existence of maximal matchings), some metric properties (radius, diameter, center, periphery, medianicity), some properties on subhypercubes (cube coefficients and polynomials, cube indices, decomposition in subhypercubes), and, finally, the distribution of the degrees.
\end{abstract}

Keywords: Hypercube, Fibonacci cube, bipartite graph, median graph, subhypercube 2000 MSC: 05C30, 05C75, 05C12, 05C31

\section{Introduction}

The hypercubes $Q_{n}$ are one of the most popular architectures for interconnection networks for multicomputers. They are highly regular, but the fact that the number of nodes grows very rapidly, as $n$ increases, limits considerably the choice of the size of the networks. To overcome disadvantages of this kind, some alternative architectures have been introduced. One of these alternatives is given by the Fibonacci cubes $\Gamma_{n}[11,12]$. These graphs can be embedded in hypercubes and efficiently emulate many hypercube algorithms.

Fibonacci cubes, however, turned out to be interesting on their own $[12,24]$. They have several interesting structural, metric, combinatorial and enumerative properties [19, 25], and many applications in theoretical chemistry [19]. Similar results can be obtained for many other families of graphs deriving from Fibonacci cubes, such as the Lucas cubes [23], the generalized Fibonacci cubes [17, 14], the extended Fibonacci cubes [31], the generalized Lucas cubes [15], the extended Lucas cubes [29], the widened Fibonacci cubes [26].

In this paper, we introduce the Pell graphs $\Pi_{n}$, a new family of graphs similar to the Fibonacci cubes. The Fibonacci cube $\Gamma_{n}$ is defined on the set of the binary strings of length $n$ with no consecutive 1's, where two vertices are adjacent whenever they differ in exactly

Email address: emanuele.munarini@polimi.it (Emanuele Munarini) 
one position (i.e. when they have Hamming distance 1). Similarly, the Pell graph $\Pi_{n}$ is defined on the set of certain strings of length $n$ on the alphabet $\{0,1,2\}$, where two vertices are adjacent whenever they differ in a suitable factor (the exact definition will be given in Section 2). The name of these graphs derives from the fact that the strings considered as vertices of $\Pi_{n}$ are enumerated by the Pell numbers, i.e. by the numbers $p_{n}$ defined by the recurrence $p_{n+2}=2 p_{n+1}+p_{n}$ with $p_{0}=1$ and $p_{1}=2$.

Pell graphs, as well as hypercubes and Fibonacci cubes, have several remarkable properties. In this paper, we obtain some structural and metric properties, and some enumerative properties on subhypercubes and the distribution of the degrees. Specifically, in Section 2 , we recall some basic definitions on graphs, formal languages and formal series. Then, we recall the definition of the Fibonacci cubes $\Gamma_{n}$ and we define the Pell graphs $\Pi_{n}$. In Section 3, we determine a recursive structure for the Pell graphs by means of a canonical decomposition and then we use such a decomposition to obtain some elementary properties, such as the bipartiteness and the existence of maximal matchings. In Section 4, we obtain some metric properties, such as the radius, the diameter, the center and the periphery. In Section 5, we show that the Pell graph $\Pi_{n}$ is a subgraph of the Fibonacci cube $\Gamma_{2 n-1}$. Then, we determine some other metric properties, such as the medianicity. In Section 6, we study some enumerative problems concerning subhypercubes. In particular, we obtain the cube coefficients (i.e. the number of the subhypercubes of a given order), the cube polynomials, the cube indices and a decomposition in hypercubes. Finally, in Section 7, we obtain the distribution of the degrees. In particular, we obtain the maximum, the minimum and the medium degree, the chromatic index, and the generating series for the degrees and the medium degrees.

\section{Basic definitions}

Here, we consider only finite undirected simple graphs. Specifically, a graph is a pair $G=(V, E)$, where $V=V(G)$ is a finite set of vertices and $E=E(G)$ is a finite set of edges consisting of unordered pairs of vertices. We write $v$ adj $w$ when the vertices $v$ and $w$ are adjacent, i.e. when they are connected by an edge, i.e. when $\{v, w\} \in E(G)$. A subgraph of $G$ is a graph $S=(X, Y)$ where $X \subseteq V(G)$ and $Y \subseteq E(G)$. In this case, we write $S \subseteq G$. If $X \subseteq V(G)$, then we denote by $\langle X\rangle$ the induced subgraph of $G$, i.e. the subgraph with vertex set $X$ and with edge set consisting of all edges of $G$ having both endpoints in $X$.

The Cartesian product of two graphs $G$ and $H$ is the graph $G \square H$ with vertex set $V(G) \times$ $V(H)$ where $\left(v_{1}, w_{1}\right)$ adj $\left(v_{2}, w_{2}\right)$ whenever $v_{1}$ adj $v_{2}$ and $w_{1}=w_{2}$, or $v_{1}=v_{2}$ and $w_{1}$ adj $w_{2}$.

As usual, $K_{n}$ denotes the complete graph on $n$ vertices. For a general reference on graph theory, see [3].

Now, we recall some basic definitions on formal languages. The free monoid over an alphabet $\mathcal{A}=\left\{\ell_{1}, \ldots, \ell_{r}\right\}$ is the set $\mathcal{A}^{*}$ of all strings (words) $\alpha=a_{1} \cdots a_{n}$ with $a_{1}, \ldots, a_{n} \in \mathcal{A}$ and $n \in \mathbb{N}$. In particular, for $n=0$, we have the empty string $\varepsilon$. The product of two strings $\alpha=a_{1} \cdots a_{m}$ and $\beta=b_{1} \cdots b_{n}$ is the concatenation $\alpha \beta=a_{1} \cdots a_{m} b_{1} \cdots b_{n}$, with the empty string as the identity element. A factor of a string $\alpha \in \mathcal{A}^{*}$ is a string $\beta \in \mathcal{A}^{*}$ such that $\alpha=\alpha_{1} \beta \alpha_{2}$, with $\alpha_{1}, \alpha_{2} \in \mathcal{A}^{*}$. If $\alpha \in \mathcal{A}^{*}$ and $S \subseteq \mathcal{A}^{*}$, then we set $\alpha S=\{\alpha \beta: \beta \in S\}$. 
Furthermore, we write $S+T$ to denote the disjoint union of two disjoint subsets $S, T \subseteq \mathcal{A}^{*}$. For instance, we have the decomposition $\mathcal{A}^{*}=\varepsilon+\ell_{1} \mathcal{A}^{*}+\cdots+\ell_{r} \mathcal{A}^{*}$. A language over a finite alphabet $\mathcal{A}$ is any subset $\mathscr{L}$ of $\mathcal{A}^{*}$. A binary string is a word over the alphabet $\mathcal{B}=\{0,1\}$. The Hamming distance between two binary strings $\alpha$ and $\beta$ is the number of bits where $\alpha$ and $\beta$ differ.

The hypercube $Q_{n}$ is the simple graph where the vertices are the binary strings of length $n$ and two vertices are adjacent when they differ in exactly one position, i.e. when they have Hamming distance equal to 1. Equivalently, we have $Q_{n}=K_{2} \square \cdots \square K_{2}$ ( $n$ times).

A Fibonacci string is a binary string with no two consecutive ones. If $\mathcal{F}_{n}$ is the set of all Fibonacci strings of length $n$, then $\mathcal{F}_{n+2}=0 \mathcal{F}_{n+1}+10 \mathcal{F}_{n}$ and $\mathcal{F}_{0}=\{\varepsilon\}, \mathcal{F}_{1}=\{0,1\}$. If $f_{n}=\left|\mathcal{F}_{n}\right|$, then we have the recurrence $f_{n+2}=f_{n+1}+f_{n}$ with the initial values $f_{0}=1$ and $f_{1}=2$. So, $f_{n}=F_{n+2}$ is a Fibonacci number [28, A000045].

The Fibonacci cube $\Gamma_{n}$, [24], is the simple graph where the vertices are the Fibonacci strings of length $n$ and two vertices are adjacent when they differ in exactly one position, i.e. when they have Hamming distance equal to 1. Fibonacci cubes have several interesting properties. For instance, we have the following decomposition property. The recurrence $\mathcal{F}_{n+2}=0 \mathcal{F}_{n+1}+10 \mathcal{F}_{n}$ for Fibonacci strings can be extended to Fibonacci cubes [12]. Indeed, the strings in $0 \mathcal{F}_{n+1}$ generate a subgraph of $\mathcal{F}_{n+2}$ isomorphic to $\Gamma_{n+1}$ and the strings in $10 \mathcal{F}_{n}$ generate a subgraph of $\mathcal{F}_{n+2}$ isomorphic to $\Gamma_{n}$. Moreover, for any string $\alpha \in \mathcal{F}_{n}, 00 \alpha$ and $10 \alpha$ are adjacent. This is the only way in which a vertex of the first component can be connected with an edge to a vertex of the second component. We write $\Gamma_{n+2}=\Gamma_{n+1} \oplus \Gamma_{n}$ to denote this decomposition.

A Pell string is a word on the alphabet $\mathcal{T}=\{0,1,2\}$ where there are no maximal blocks of 2's of odd length. Equivalently, a Pell string is an arbitrary word on the alphabet $\mathcal{T}^{\prime}=\{0,1,22\}$. Let $\mathcal{P}_{n}$ be the set of all Pell strings of length $n$ and let $P_{n}=\left|\mathcal{P}_{n}\right|$. Since $\mathcal{P}_{n+2}=0 \mathcal{P}_{n+1}+1 \mathcal{P}_{n+1}+22 \mathcal{P}_{n}$ and $\mathcal{P}_{0}=\{\varepsilon\}$ and $\mathcal{P}_{1}=\{0,1\}$, we have the recurrence $P_{n+2}=2 P_{n+1}+P_{n}$ with the initial values $P_{0}=1$ and $P_{1}=2$. So $P_{n}=p_{n}$ and the Pell strings of length $n$ are counted by the Pell number $p_{n}$. These numbers form sequence A000129 in [28] and their first values are 1, 2, 5, 12, 29, 70, 169, 408, 985, 2378, 5741, 13860, 33461.

The Pell graph $\Pi_{n}$ is the simple graph where the vertices are the Pell strings of length $n$ and where two vertices are adjacent whenever one of them can be obtained from the other by replacing a 0 with a 1 (or viceversa), or by replacing a factor 11 with 22 (or viceversa). We have $\Pi_{0}=K_{1}, \Pi_{1}=K_{2}$. See Figure 1 for $\Pi_{2}, \Pi_{3}$ and $\Pi_{4}$.

Let $\mathscr{L}$ be a language over a finite alphabet $\mathcal{A}$, i.e. $\mathscr{L} \subseteq \mathcal{A}^{*}$. A statistic on $\mathscr{L}$ is a map $\sigma: \mathscr{L} \rightarrow \mathbb{N}$. For instance, the length $|\alpha|$ of a string $\alpha$ is a statistic on $\mathscr{L}$. Given a language $\mathscr{L}$, endowed with a statistic $\sigma$, we can consider the generating series

$$
F(x, t)=\sum_{\alpha \in \mathscr{L}} x^{\sigma(\alpha)} t^{|\alpha|}=\sum_{n, k \geq 0} F_{n, k} x^{k} t^{n}
$$

where $x$ marks the value of the statistic, $t$ marks the length and $F_{n, k}$ is the number of strings $\alpha \in \mathscr{L}$ with length $n$ and $\sigma(\alpha)=k$. For more details on languages and formal series, see [8]. 


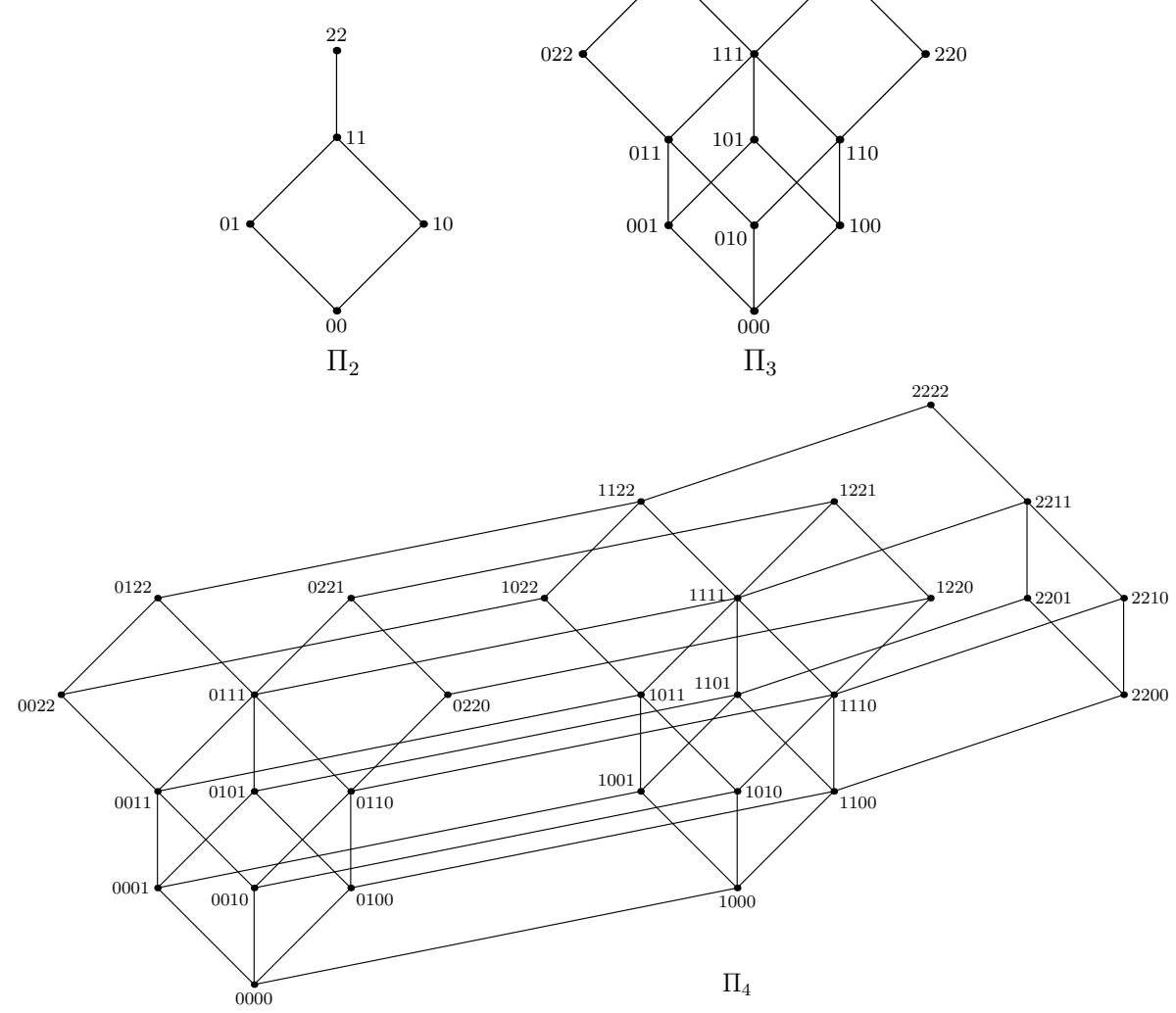

Figure 1: The Pell graphs $\Pi_{2}, \Pi_{3}$ and $\Pi_{4}$.

A Riordan matrix [27] is an infinite lower triangular matrix $R=\left[r_{n, k}\right]_{n, k \geq 0}=(g(t), f(t))$ whose columns have generating series

$$
r_{k}(t)=\sum_{n \geq k} r_{n, k} t^{n}=g(t) f(t)^{k},
$$

where $g(t)=\sum_{n \geq 0} g_{n} t^{n}$ and $f(t)=\sum_{n \geq 0} f_{n} t^{n}$ are formal series with $g_{0}=1, f_{0}=0$ and $f_{1} \neq 0$. The associated row polynomials are the polynomials defined by $R_{n}(x)=\sum_{k=0}^{n} r_{n, k} x^{k}$.

For instance, the Delannoy numbers $D_{n, k},[7$, p. 81] [28, A008288], are defined as the entries of the Riordan matrix

$$
D=\left[D_{n, k}\right]_{n, k \geq 0}=\left(\frac{1}{1-t}, \frac{t+t^{2}}{1-t}\right) .
$$

This means that we have the generating series

$$
\sum_{n \geq k} D_{n, k} t^{n}=\frac{1}{1-t}\left(\frac{t+t^{2}}{1-t}\right)^{k}=\frac{\left(t+t^{2}\right)^{k}}{(1-t)^{k+1}} .
$$


The row polynomials of $D$ are the Delannoy polynomials $d_{n}(x)=\sum_{k=0}^{n} D_{n, k} x^{k}$, with generating series

$$
\sum_{n \geq 0} d_{n}(x) t^{n}=\sum_{n, k \geq 0} D_{n, k} x^{k} t^{n}=\frac{1}{1-(1+x) t-x t^{2}} .
$$

The row-sums of the matrix $D$ are the Pell numbers, i.e. $\sum_{k=0}^{n} D_{n, k}=p_{n}$.

The incremental ratio of a formal series $f(t)=\sum_{n \geq 0} f_{n} t^{n}$ is defined by

$$
\mathcal{R} f(t)=\sum_{n \geq 0} f_{n+1} t^{n}=\frac{f(t)-f_{0}}{t} .
$$

In particular, if we apply this operator two times, then we have

$$
\mathcal{R}^{2} f(t)=\mathcal{R}(\mathcal{R} f(t))=\sum_{n \geq 0} f_{n+2} t^{n}=\frac{f(t)-f_{0}-f_{1} t}{t^{2}}
$$

\section{Canonical decomposition}

As Fibonacci cubes, also Pell graphs admit a recursive decomposition. Indeed, we have $\mathcal{P}_{n+2}=0 \mathcal{P}_{n+1}+1 \mathcal{P}_{n+1}+22 \mathcal{P}_{n}$. The strings in $0 \mathcal{P}_{n+1}$ generate a graph isomorphic to $\Pi_{n+1}$. Similarly, the strings in $1 \mathcal{P}_{n+1}$ generate a graph isomorphic to $\Pi_{n+1}$. Finally, the strings in $22 \mathcal{P}_{n}$ generate a graph isomorphic to $\Pi_{n}$. Every string $0 \alpha$ in $0 \mathcal{P}_{n+1}$ is adjacent to the string $1 \alpha$ in $1 \mathcal{P}_{n+1}$. Every string $11 \alpha$ in $11 \mathcal{P}_{n} \subseteq 1 \mathcal{P}_{n+1}$ is adjacent to the string $22 \alpha$ in $22 \mathcal{P}_{n}$. The strings in $0 \mathcal{P}_{n+1}$ are never adjacent to the strings in $22 \mathcal{P}_{n}$. Then $\Pi_{n+2}$ decomposes into two disjoint copies of $\Pi_{n+1}$ and a copy of $\Pi_{n}$. We say that this is the canonical decomposition of $\Pi_{n+2}$ and we write $\Pi_{n+2}=\Pi_{n+1} \oplus \Pi_{n+1} \oplus \Pi_{n}$. See Figure 2 for a schematic representation of such a decomposition and see Figure 3(a) for a concrete example.

Clearly, the canonical decomposition is not the unique recursive decomposition. Indeed, a similar decomposition can be obtained by the identity $\mathcal{P}_{n+2}=\mathcal{P}_{n+1} 0+\mathcal{P}_{n+1} 1+\mathcal{P}_{n} 22$. Notice that this is due also to the fact that the Pell graph $\Pi_{n}$ is symmetric (see Figure 3 ). Indeed, for every Pell string $\alpha=a_{1} a_{2} \cdots a_{n}$, the reverse string $\alpha^{*}=a_{n} \cdots a_{2} a_{1}$ is still a Pell string and adjacency is preserved under this operation.

Notice that the strings in $0 \mathcal{P}_{n+1} \cup 1 \mathcal{P}_{n+1}$ generate a subgraph of $\Pi_{n+2}$ isomorphic to $K_{2} \square \Pi_{n+1}$. So, we also have the decomposition $\Pi_{n+2}=\left(K_{2} \square \Pi_{n+1}\right) \oplus \Pi_{n}$. This is the second form of the canonical decomposition. Notice also that $\left\langle 11 \mathcal{P}_{n} \cup 22 \mathcal{P}_{n}\right\rangle=K_{2} \square \Pi_{n}$. See again Figure 2.

Several properties of the Pell graphs can be obtained by their canonical decomposition, as we will see in the next two propositions and in the following of the paper.

An independent set of a graph $G$ is a subset $X \subseteq V(G)$ with no adjacent vertices. A graph $G$ is bipartite when the vertex set can be decomposed into two disjoint non-empty independent subsets $V_{1}$ and $V_{2}$. In this case, the pair $\left(V_{1}, V_{2}\right)$ is a bipartition of $G$. A proper 2-coloring of a graph $G$ is a map $\chi: V(G) \rightarrow\{0,1\}$ satisfying the property: if $v, w \in V(G)$ and $v$ adj $w$, then $\chi(v) \neq \chi(w)$. A graph is bipartite if and only if admits a proper 2coloring. In this case, the bipartition is $\left(V_{0}, V_{1}\right)$, where $V_{k}=\{v \in V(G): \chi(v)=k\}$, for $k=0,1$. 


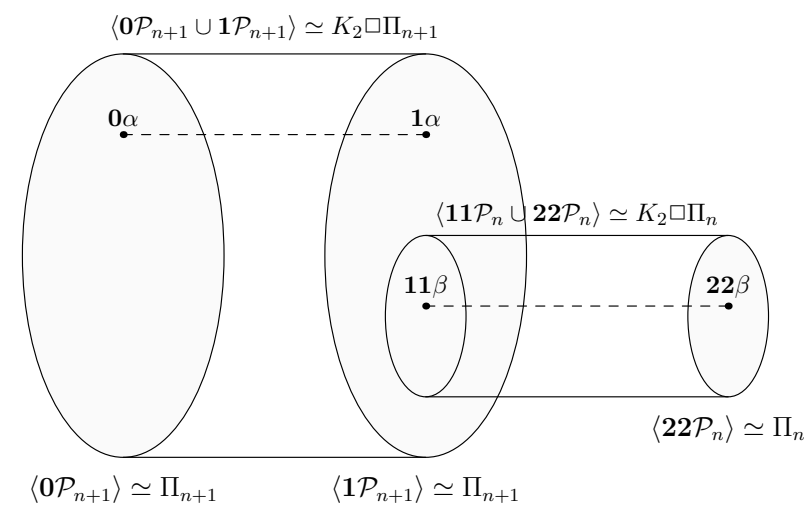

Figure 2: Canonical decomposition: $\Pi_{n+2}=\Pi_{n+1} \oplus \Pi_{n+1} \oplus \Pi_{n}$.

Proposition 1. For every $n \in \mathbb{N}$, the Pell graph $\Pi_{n}$ is bipartite.

Proof. We proceed by induction on $n$. First, we have that $\Pi_{0}=K_{1}$ and $\Pi_{1}=K_{2}$ are bipartite. Now, we assume that $\Pi_{k}$ is bipartite for every $k \leq n+1$ and we prove that this is true also for $\Pi_{n+2}$. By the canonical decomposition, we have $\Pi_{n+2}=\Pi_{n+1} \oplus \Pi_{n+1} \oplus$ $\Pi_{n}$. We choose a proper 2-coloring $\chi$ of the first component $\Pi_{n+1}$. Then, we consider the complementary proper coloring $\chi^{\prime}=1-\chi$ for the second component $\Pi_{n+1}$. The coloring $\chi^{\prime}$ induces a proper 2-coloring of the subgraph isomorphic to $\Pi_{n}$ contained in the second component $\Pi_{n+1}$. Finally, we consider the coloring $\chi$ restricted to the third component $\Pi_{n}$. In this way, we obtain a proper 2-coloring of the whole $\Pi_{n+2}$.

REMARK 2. Theorem 1 can also be proved directly. Indeed, we can define an order relation on the set $\mathcal{P}_{n}$ of Pell strings of length $n$. For every $\alpha=a_{1} \cdots a_{n}$ and $\beta=b_{1} \cdots b_{n}$ in $\mathcal{P}_{n}$, we set $\alpha \leq \beta$ whenever $a_{i} \leq b_{i}$, for all $i \in\{1,2, \ldots, n\}$. The poset $\mathscr{P}_{n}=\left\langle\mathcal{P}_{n}, \leq\right\rangle$ has a minimum $\widehat{0}=00 \cdots 0$ and an element $\alpha$ is covered by $\beta$ if and only if $\alpha=\xi_{1} 0 \xi_{2}$ and $\beta=\xi_{1} 1 \xi_{2}$ or $\alpha=\xi_{1} 11 \xi_{2}$ and $\beta=\xi_{1} 22 \xi_{2}$. The Hasse diagram of $\mathscr{P}_{n}$ is the Pell graph $\Pi_{n}$. Moreover, $\mathscr{P}_{n}$ is ranked and the rank function is defined by $r(\alpha)=\omega_{1}(\alpha)+\frac{3}{2} \omega_{2}(\alpha)$, where $\omega_{x}(\alpha)$ denotes the number of occurrences of the letter $x$ in the string $\alpha$. Indeed, $r(\widehat{0})=0$ and $\alpha$ is covered by $\beta$ if and only if $r(\beta)=r(\alpha)+1$. So, $\Pi_{n}$ is a bipartite graph, with bipartition $\left(E_{n}, O_{n}\right)$ where $E_{n}$ is the set of all vertices with even rank and $O_{n}$ is the set of all vertices with odd rank. In particular, the map $\chi$, defined by $\chi(\alpha)=r(\alpha) \bmod 2$, is a proper 2-coloring of $\Pi_{n}$.

A matching of a graph $G$ is a set of independent edges. A perfect matching, or 1-factor, is a matching covering all vertices of $G$. A semi-perfect matching, or near 1-factor, is a matching covering all vertices of $G$ except one.

Proposition 3. For every $n \in \mathbb{N}, \Pi_{2 n+1}$ admits a perfect matching and $\Pi_{2 n}$ admits a semiperfect matching.

Proof. We proceed by induction on $n$. First, we have that $\Pi_{1}=K_{2}$ has exactly one perfect matching. Now, we assume that $\Pi_{2 n+1}$ has a perfect matching and we prove that this is true 


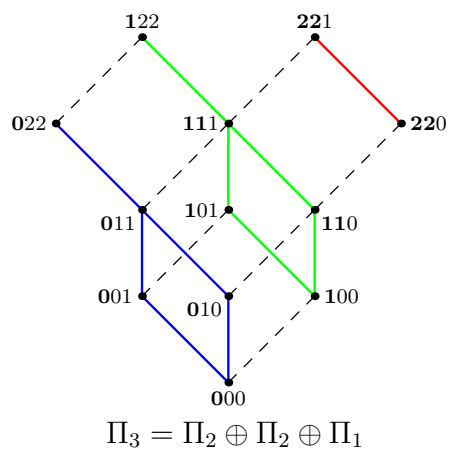

(a)

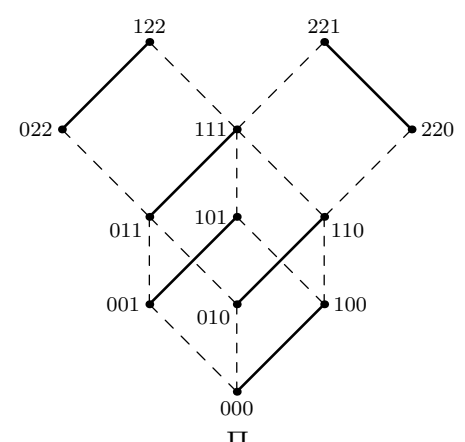

(b)

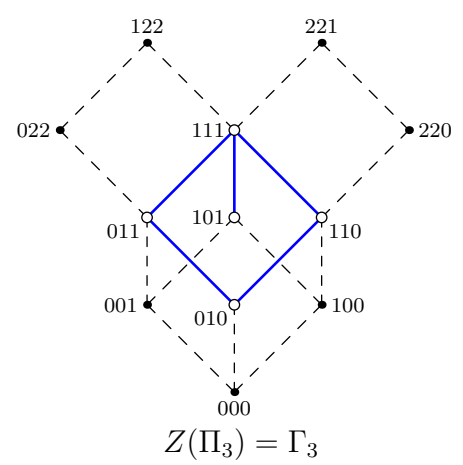

(c)

Figure 3: (a) canonical decomposition of the Pell graph $\Pi_{3}$, (b) a perfect matching of $\Pi_{3}$, (c) the subgraph induced by the center of $\Pi_{3}$, isomorphic to $\Gamma_{3}$.

also for $\Pi_{2 n+3}$. By the canonical decomposition, we have $\Pi_{2 n+3}=\left(K_{2} \square \Pi_{2 n+2}\right) \oplus \Pi_{2 n+1}$. The first component $K_{2} \square \Pi_{2 n+2}$ admits a perfect matching consisting of the edges connecting a vertex $0 \alpha$ with the corresponding vertex $1 \alpha$. The second component $\Pi_{2 n+1}$ has a perfect matching, by hypothesis. The union of these two matchings is a perfect matching of $\Pi_{2 n+3}$. See Figure 3(b) for an example. In a similar way, we can prove that $\Pi_{2 n}$ has a semi-perfect matching.

\section{Basic metric properties}

We recall some definitions for a connected simple graph $G[5,6]$. The distance $d(v, w)$ between two vertices $v$ and $w$ of $G$ is the length of a shortest path connecting them. The eccentricity $e(v)$ of a vertex $v$ of $G$ is the distance to a node farthest from $v$, i.e. $e(v)=$ $\max _{u \in V(G)} d(u, v)$. The radius $\operatorname{rad}(G)$ of $G$ is the smallest of its eccentricities, and the $d i$ ameter $\operatorname{diam}(G)$ is the largest, i.e. $\operatorname{rad}(G)=\min _{v \in V(G)} e(v)$ and $\operatorname{diam}(G)=\max _{v \in V(G)} e(v)$. A vertex $v$ is central when $e(v)=\operatorname{rad}(G)$ and is peripheral when $e(v)=\operatorname{diam}(G)$. The center $Z(G)$ is the set of all central vertices of $G$, and the periphery $P(G)$ is the set of all peripheral vertices of $G$.

Proposition 4. For every $n \in \mathbb{N}$, we have $\operatorname{rad}\left(\Pi_{n}\right)=n$ and $\operatorname{diam}\left(\Pi_{n}\right)=n+\left\lfloor\frac{n}{2}\right\rfloor$.

Proof. First, we notice that $e\left(1^{n}\right)=n$. Indeed, since we can change a 1 in 0 or a pair 11 in 22 the maximum distance can be reached changing each 1 in 0 . Now, we prove that $e(\alpha) \geq n$, for every Pell string $\alpha \in \mathcal{P}_{n}$. Suppose that $\alpha$ contains $2 k$ letters 2 , and consequently $n-2 k$ letters 0 or 1 . A single letter 0 (or 1 ) can be changed to 1 (or 0 ) with a single move. A factor 22 can be changed to 00, with 3 moves, for instance $22 \rightsquigarrow 11 \rightsquigarrow 01 \rightsquigarrow 00$. So $e(\alpha) \geq(n-2 k)+3 k=n+k \geq n$. Hence, the minimum eccentricity is $n$, i.e. $\operatorname{rad}\left(\Pi_{n}\right)=n$.

To have a vertex with maximum eccentricity we need a string with a maximum number of factors 22. So, if $n=2 m$, we have the string $(22)^{m}$ with eccentricity $3 m=n+n / 2$. If $n=2 m+1$, we have the string $(22)^{m} 1$ with eccentricity $3 m+1=n+\lfloor n / 2\rfloor$. So, the maximum eccentricity is $n+\lfloor n / 2\rfloor$, i.e. $\operatorname{diam}\left(\Pi_{n}\right)=n+\lfloor n / 2\rfloor$. 
Proposition 5. For every $n \in \mathbb{N}$, the subgraph of $\Pi_{n}$ induced by the center $Z\left(\Pi_{n}\right)$ is isomorphic to the Fibonacci cube $\Gamma_{n}$.

Proof. If $\alpha \in \mathcal{P}_{n}$ has $2 k$ letters 2 , with $k>0$, then $e(\alpha) \geq n+k>n$ and $\alpha \notin Z\left(\Pi_{n}\right)$. Moreover, if $\alpha \in \mathcal{P}_{n}$ is a binary string with at least one factor 00 (which can be transformed to 22 in three moves), then $e(\alpha) \geq n+1>n$ and $\alpha \notin Z\left(\Pi_{n}\right)$. So, if $\alpha \in Z\left(\Pi_{n}\right)$, then $\alpha$ is a binary strings with no adjacent 0 's, i.e. it is equivalent to a Fibonacci string. To obtain a string at maximum distance from a string of this kind, we can only change a single letter 0 (or 1 ) in 1 (or 0 ) with a single move. So, their eccentricity is at most $n$, and consequently is exactly $n$. Then $Z\left(\Pi_{n}\right) \simeq \mathcal{F}_{n}$. Moreover, two strings in $Z\left(\Pi_{n}\right)$ are adjacent when they differ exactly in one position. This means that $\left\langle Z\left(\Pi_{n}\right)\right\rangle \simeq \Gamma_{n}$. See Figure $3(\mathrm{c})$ for an example.

Proposition 6. For $n=2 m$, the periphery $P\left(\Pi_{n}\right)$ consists of the $2^{m}$ strings $Y_{1} Y_{2} \cdots Y_{m}$ where $Y_{i} \in\{00,22\}$ for every $i \in\{1,2, \ldots, m\}$. For $n=2 m+1$, the periphery $P\left(\Pi_{n}\right)$ consists of the $(m+1) 2^{m+1}$ strings $X Y_{1} Y_{2} \cdots Y_{m-1} Y_{m}, Y_{1} X Y_{2} \cdots Y_{m-1} Y_{m}, \ldots, Y_{1} Y_{2} \cdots Y_{m-1} X Y_{m}$ and $Y_{1} Y_{2} \cdots Y_{m-1} Y_{m} X$ where $X \in\{0,1\}$ and $Y_{i} \in\{00,22\}$ for every $i \in\{1,2, \ldots, m\}$.

Proof. A peripheral vertex of $\Pi_{n}$ is a string $\alpha$ with $e(\alpha)=\operatorname{diam}\left(\Pi_{n}\right)$, and the maximum distance can be reached when $\alpha$ contains the maximum number of factors 00 or 22 .

\section{Pell binary strings}

Since all binary strings are Pell strings, the binary strings of length $n$ generate a subgraph of $\Pi_{n}$ isomorphic to $Q_{n}$. So, we have the inclusions $\Gamma_{n} \subseteq Q_{n} \subseteq \Pi_{n}$. Moreover, the Pell graph $\Pi_{n}$ can be viewed as a subgraph of a Fibonacci cube, as proved in the next theorem.

Theorem 7. For $n \geq 1$, we have the inclusion $\Pi_{n} \subseteq \Gamma_{2 n-1}$.

Proof. Every Pell string decomposes uniquely in the product of the factors 0,1 and 22 . This allows us to define the map $\psi: \mathcal{P}_{n} \rightarrow \mathcal{F}_{2 n}$ by setting $\psi(0)=10, \psi(1)=00$ and $\psi(22)=$ 0100. For instance, $\psi(122010022221)=\psi(1) \psi(22) \psi(0) \psi(1) \psi(0) \psi(0) \psi(22) \psi(22) \psi(1)=$ 000100100010100100010000 . Notice that $\psi(\alpha)$ is a Fibonacci string of length $2 n$ ending with 0 (for $n \geq 1$ ). Let $n \geq 1$ and define the map $\psi: \Pi_{n} \rightarrow \Gamma_{2 n-1}$ as before, except for the fact that the last 0 is removed. $\psi$ is a graph map, i.e. preserves adjacency. Let $\alpha$ adj $\beta$. If $\alpha=\xi_{1} 0 \xi_{2}$ and $\beta=\xi_{1} 1 \xi_{2}$, then $\psi(\alpha)=\psi\left(\xi_{1}\right) 10 \psi\left(\xi_{2}\right)$ and $\psi(\beta)=\psi\left(\xi_{1}\right) 00 \psi\left(\xi_{2}\right)$, and so $\psi(\alpha)$ adj $\psi(\beta)$. If $\alpha=\xi_{1} 11 \xi_{2}$ and $\beta=\xi_{1} 22 \xi_{2}$, then $\psi(\alpha)=\psi\left(\xi_{1}\right) 0000 \psi\left(\xi_{2}\right)$ and $\psi(\beta)=\psi\left(\xi_{1}\right) 0100 \psi\left(\xi_{2}\right)$, and again $\psi(\alpha)$ adj $\psi(\beta)$. Finally, $\psi$ is an injective map. So, in conclusion, the Fibonacci cube $\Gamma_{2 n-1}$ contains a subgraph isomorphic to the Pell graph $\Pi_{n}$.

The binary strings in $\psi\left(\mathcal{P}_{n}\right) \subseteq \mathcal{F}_{2 n}$ will be called Pell binary strings. Let $\mathcal{F}_{2 n}^{*}$ be the set of Fibonacci strings of length $2 n$ with no factors 0101 and without a final 1 . Let $\Gamma_{2 n}^{*}$ be the Hamming graph generated by $\mathcal{F}_{2 n}^{*}$.

Theorem 8. The graphs $\Pi_{n}$ and $\Gamma_{2 n}^{*}$ are isomorphic. 
Proof. Any string $\alpha \in \mathcal{F}_{2 n}^{*}$ decomposes uniquely as a product of 00,10 and 01 . Since we cannot have the factors 0110 and 0101, and since 1 cannot be in final position, then 01 is necessarily followed by 00 . Hence, $\alpha$ decomposes uniquely as a product of 00, 10 and 0100 , and this means that $\mathcal{F}_{2 n}^{*}=\psi\left(\mathcal{P}_{n}\right)$. By Theorem 7 , we have the claim.

A median of three vertices of a connected graph is a vertex that lies simultaneously on geodesics between any two of them. A graph $G$ is a median graph when every triple of (not necessarily distinct) vertices of $G$ has a unique median [9, 13]. Median graphs are characterized as retracts of hypercubes [13, p. 76]. All hypercubes $Q_{n}$ are median. Furhtermore, S. Klavžar proved [18] that Fibonacci and Lucas cubes are median graphs by using the following property due to Mulder.

Theorem 9 (Mulder [22]). A connected graph $G$ is a median graph if and only if it is an induced graph of an $n$-cube such that for any three vertices of $G$ their median in the $n$-cube is also a vertex of $G$.

Such a proof can be extended to Pell graphs, as proved in the next Theorem.

Theorem 10. For any $n \in \mathbb{N}$, the Pell graph $\Pi_{n}$ is median.

Proof. By Theorem 8, the Pell graph $\Pi_{n}$ is isomorphic to the graph $\Gamma_{2 n}^{*}$, which is an induced subgraph of $Q_{2 n}$. We prove that $\Gamma_{2 n}^{*}$ is a median closed subgraph of $Q_{2 n}$. Let $\alpha=a_{1} \cdots a_{2 n}$, $\beta=b_{1} \cdots b_{2 n}$ and $\gamma=c_{1} \cdots c_{2 n}$ be three arbitrary vertices of $\Gamma_{2 n}^{*}$ and let $\mu=m_{1} \cdots m_{2 n}$ be their median in $Q_{2 n}$. Then $\mu$ is obtained by the majority rule, which says that the $i$-th coordinate of $\mu$ is equal to the element which appears at least twice among the $i$-th coordinates $a_{i}, b_{i}, c_{i}$. We write $m_{i}=\operatorname{maj}\left(a_{i}, b_{i}, c_{i}\right)$. First, we have that $\mu$ is a Fibonacci string [18]. Indeed, if $m_{i}=1$ and $m_{i+1}=1$, i.e. $\operatorname{maj}\left(a_{i}, b_{i}, c_{i}\right)=1$ and $\operatorname{maj}\left(a_{i+1}, b_{i+1}, c_{i+1}\right)=1$, then in at least one of the vertices $\alpha, \beta$ and $\gamma$ there are two consecutive 1's, which is impossible. Moreover, in $\mu$ there are no substrings 0101. Indeed, if $\mu_{2 i+1}=1$ and $\mu_{2 i+3}=1$, then (as before) in at least one of the vertices $\alpha, \beta$ and $\gamma$ there is a substring 0101, which is impossible. Finally, since $a_{2 n}=b_{2 n}=c_{2 n}=0$, we have $m_{2 n}=0$. In conclusion, $\Gamma_{2 n}^{*}$ is a median closed subgraph of $Q_{2 n}$ and consequently is a median graph by Mulder's Theorem 9 .

Theorem 11. The Pell graph $\Pi_{n}$ is an isometric subgraph of the hypercube $Q_{2 n-1}(n \geq 1)$.

Proof. By Theorem 10, we have that $\Pi_{n}$ is a retract of the hypercube $Q_{2 n-1}$. Since any retract of a graph $G$ is an isometric subgraph of $\mathrm{G}$, we have the claim.

REMARK 12. The number of Pell binary strings of length $2 n$ with $k 1$ 's is the Delannoy number $D_{n, k}$. Indeed, if $\mathcal{D}$ is the set of all Pell binary strings and $D(x, t)$ is the associated generating series, where $x$ marks the number of 1 's and $t$ the semi-length, then we have the decomposition $\mathcal{D}=\varepsilon+10 \mathcal{D}+00 \mathcal{D}+0100 \mathcal{D}$ and consequently the equation

$$
D(x, t)=1+x t D(x, t)+t D(x, t)+x t^{2} D(x, t)
$$

whose solution is the generating series (1) of the Delannoy numbers. 


\section{Subhypercubes}

Given a graph $G$, the cube coefficient $q_{k}(G)$ of $G$ is the number of subgraphs of $G$ isomorphic to the hypercube $Q_{k}$. In particular, $q_{0}(G)$ is the number of vertices of $G, q_{1}(G)$ is the number of edges of $G$ and $q_{2}(G)$ is the number of 4-cycles of $G$. The cube polynomial of $G$ is defined [4, 20] by

$$
q(G ; x)=\sum_{k \geq 0} q_{k}(G) x^{k} .
$$

The cube number $q(G)$ of $G$ is the total number of subhypercubes, i.e. $q(G)=q(G ; 1)=$ $\sum_{k \geq 0} q_{k}(G)$. For simplicity, let $q_{n, k}=q_{k}\left(\Pi_{n}\right), q_{n}(x)=q\left(\Pi_{n} ; x\right)$ and $q_{n}=q\left(\Pi_{n}\right)$.

\subsection{Cube coefficients}

Let $\delta_{m, n}$ be the Kronecker delta, defined, as usual, by $\delta_{m, n}=1$ when $m=n$ and $\delta_{m, n}=0$ otherwise.

Theorem 13. The numbers $q_{n, k}$ satisfy the recurrence

$$
q_{n+2, k+1}=2 q_{n+1, k+1}+q_{n, k+1}+q_{n+1, k}+q_{n, k}
$$

with the initial values $q_{n, 0}=p_{n}, q_{0, k}=\delta_{k, 0}$ and $q_{1, k}=2 \delta_{k, 0}+\delta_{k, 1}$. Moreover, we have the generating series

$$
Q_{k}(t)=\sum_{n \geq 0} q_{n, k} t^{n}=\frac{\left(t+t^{2}\right)^{k}}{\left(1-2 t-t^{2}\right)^{k+1}}
$$

and

$$
Q(x, t)=\sum_{n, k \geq 0} q_{n, k} x^{k} t^{n}=\frac{1}{1-(2+x) t-(1+x) t^{2}} .
$$

Proof. Consider a subgraph $S$ of $\Pi_{n+2}$ isomorphic to $Q_{k+1}$, and consider the canonical decomposition $\Pi_{n+2}=\Pi_{n+1} \oplus \Pi_{n+1} \oplus \Pi_{n}=\left(K_{2} \square \Pi_{n+1}\right) \oplus \Pi_{n}$. We have the following possibilities.

1. $S$ is contained in one of the two copies of $\Pi_{n+1}$ or in the copy of $\Pi_{n}$.

2. $S=K_{2} \square Q_{k}$ is contained in $K_{2} \square \Pi_{n+1}$, with the first copy of $Q_{k}$ contained in $\Pi_{n+1}=$ $\left\langle 0 \mathcal{P}_{n+1}\right\rangle$ and the second copy of $Q_{k}$ contained in $\Pi_{n+1}=\left\langle 1 \mathcal{P}_{n+1}\right\rangle$.

3. $S=K_{2} \square Q_{k}$ is contained in $K_{2} \square \Pi_{n}$, with the first copy of $Q_{k}$ contained in $\Pi_{n}=\left\langle 11 \mathcal{P}_{n}\right\rangle$ and the second copy of $Q_{k}$ contained in $\Pi_{n}=\left\langle 22 \mathcal{P}_{n}\right\rangle$.

This analysis immediately implies recurrence (4). Now, consider the series $Q_{k}(t)=\sum_{n \geq 0} q_{n, k} t^{n}$. From recurrence (4), we have the equation

$$
\mathcal{R}^{2} Q_{k+1}(t)=2 \mathcal{R} Q_{k+1}(t)+Q_{k+1}(t)+\mathcal{R} Q_{k}(t)+Q_{k}(t) .
$$

By identities (2) and (3), this equation becomes

$$
\frac{Q_{k+1}(t)-q_{0, k+1}-q_{1, k+1} t}{t^{2}}=2 \frac{Q_{k+1}(t)-q_{0, k+1}}{t_{10}}+Q_{k+1}(t)+\frac{Q_{k}(t)-q_{0, k}}{t}+Q_{k}(t) .
$$


Since we have the initial conditions $q_{0, k}=\delta_{k, 0}$ and $q_{1, k}=2 \delta_{k, 0}+\delta_{k, 1}$, the above equation simplifies in

$$
Q_{k+1}(t)-\delta_{k, 0} t=2 t Q_{k+1}(t)+t^{2} Q_{k+1}(t)+t Q_{k}(t)-\delta_{k, 0} t+t^{2} Q_{k}(t),
$$

that is

$$
\left(1-2 t-t^{2}\right) Q_{k+1}(t)=\left(t+t^{2}\right) Q_{k}(t)
$$

Hence, we have

$$
Q_{k+1}(t)=\frac{t+t^{2}}{1-2 t-t^{2}} Q_{k}(t)
$$

and consequently

$$
Q_{k}(t)=\left(\frac{t+t^{2}}{1-2 t-t^{2}}\right)^{k} Q_{0}(t) .
$$

Since $q_{n, 0}=p_{n}$, we have $Q_{0}(t)=p(t)=\frac{1}{1-2 t-t^{2}}$ and then we have series (5). From this identity we obtain straightforwardly series $(6)$.

REMARK 14. The first values of the cube coefficients are reported in the matrix

$$
Q=\left[q_{n, k}\right]_{n, k \geq 0}=\left[\begin{array}{cccccccccc}
1 & & & & & & & & \\
2 & 1 & & & & & & & \\
5 & 5 & 1 & & & & & & \\
12 & 18 & 8 & 1 & & & & & \\
29 & 58 & 40 & 11 & 1 & & & & \\
70 & 175 & 164 & 71 & 14 & 1 & & \\
169 & 507 & 601 & 357 & 111 & 17 & 1 & \\
408 & 1428 & 2048 & 1550 & 664 & 160 & 20 & 1 & \\
985 & 3940 & 6632 & 6106 & 3346 & 1112 & 218 & 23 & 1 \\
\cdots & & & & & & & &
\end{array}\right] .
$$

Notice that, being $Q(-1, t)=(1-t)^{-1}$, we have the identity

$$
\sum_{k \geq 0}(-1)^{k} q_{n, k}=1
$$

So, the difference between the number of subhypercubes with even order and the number of subhypercubes with odd order is exactly 1.

In particular, for the number $\ell_{n}$ of edges, whose first few values are: $0,1,5,18,58,175$, 507, 1428, 3940, 10701, 28705, 76230, 200766, we have

Proposition 15. The numbers $\ell_{n}$ satisfy the recurrence

$$
\ell_{n+2}=2 \ell_{n+1}+\ell_{n}+p_{n+1}+p_{n}
$$


with the initial values $\ell_{0}=0$ and $\ell_{1}=1$. Moreover, they have generating series

$$
\ell(t)=\sum_{n \geq 0} \ell_{n} t^{n}=\frac{t+t^{2}}{\left(1-2 t-t^{2}\right)^{2}}=\frac{1}{2} t p^{\prime}(t)=\frac{1}{2} \sum_{n \geq 0} n p_{n} t^{n}
$$

and consequently

$$
\ell_{n}=\frac{n}{2} p_{n}
$$

Proof. Since $q_{n, 0}=p_{n}$ and $q_{n, 1}=\ell_{n}$, recurrence (4) with $k=0$ reduces to recurrence (7) and series (5) reduces to series (8).

Similarly, for the numbers $c_{n}=q_{n, 2}$ of 4-cycles, whose first few values are: $0,0,1,8,40$, $164,601,2048,6632,20680,62633,185352,538272$, it is possible to prove

Proposition 16. The numbers $c_{n}$ satisfy the recurrence $c_{n+2}=2 c_{n+1}+c_{n}+\ell_{n+1}+\ell_{n}$ with the initial values $c_{0}=c_{1}=0$, and can be expressed as

$$
c_{n}=\frac{1}{16}\left(n(2 n-3) p_{n}+(n+1) p_{n-1}\right) .
$$

6.2. Cube polynomials and cube numbers

The subhypercubes of $\Pi_{n}$ can be represented as the strings of the language $\mathcal{Q}$ over the alphabet $\mathcal{H}=\{0,1,2, X, Y\}$ where the maximal blocks of 2's and the maximal blocks of $Y$ 's are of even length. The factors $X$ and $Y Y$ have to be considered as variables: $X \in\{0,1\}$ and $Y Y \in\{11,22\}$. For instance, the string $221 X 0 Y Y 1$ generates the square with vertices 22100111, 22110111, 22110221 and 22100221. Every string $\alpha \in \mathcal{Q}$ admits a standard decomposition $\alpha=\xi_{1} \xi_{2} \cdots \xi_{k}$, where each factor $\xi_{i} \in\{0,1,22, X, Y Y\}$. The order of the corresponding subhypercube is given by the number of factors $X$ and $Y Y$ in this decomposition. Moreover, we have the equation

$$
\mathcal{Q}=\varepsilon+0 \mathcal{Q}+1 \mathcal{Q}+22 \mathcal{Q}+X \mathcal{Q}+Y Y \mathcal{Q} .
$$

Theorem 17. The cube polynomials $q_{n}(x)$ satisfy the recurrence

$$
q_{n+2}(x)=(2+x) q_{n+1}(x)+(1+x) q_{n}(x)
$$

with the initial values $q_{0}(x)=1$ and $q_{1}(x)=2+x$. Moreover, they have the generating series

$$
Q(x, t)=\sum_{n \geq 0} q_{n}(x) t^{n}=\frac{1}{1-(2+x) t-(1+x) t^{2}}
$$

and can be expressed in terms of the Delannoy polynomials:

$$
q_{n}(x)=d_{n}(1+x)=\sum_{k=0}^{n} D_{n, k}(1+x)^{k} .
$$

Consequently, we have

$$
q_{n, k}=\sum_{i=k}^{n}\left(\begin{array}{l}
i \\
k
\end{array}\right) D_{n, i}=\sum_{i=k}^{n}\left(\begin{array}{l}
i \\
k
\end{array}\right) \sum_{j=0}^{n}\left(\begin{array}{l}
i \\
j
\end{array}\right)\left(\begin{array}{c}
n-j \\
i
\end{array}\right) .
$$


Proof. Let $Q(x, t)=\sum_{n, k \geq 0} q_{n, k} x^{k} t^{n}$ be the generating series of $\mathcal{Q}$, where $x$ marks the numbers of the factors $X$ and $Y Y$ in the standard decomposition (giving the order of the corresponding subhypercube) and $t$ marks the length. Then, by identity (10), we have the equation

$$
Q(x, t)=1+t Q(x, t)+t Q(x, t)+t^{2} Q(x, t)+x t Q(x, t)+x t^{2} Q(x, t)
$$

or

$$
\left(1-(2+x) t-(1+x) t^{2}\right) Q(x, t)=1
$$

from which we have generating series (12). From series (1) and (12), we have at once identity (13).

Proposition 18. The matrix $Q=\left[q_{n, k}\right]_{n, k \in \mathbb{N}}$ generated by the cube coefficients is a Riordan matrix. More precisely, we have

$$
Q=\left(\frac{1}{1-2 t-t^{2}}, \frac{t+t^{2}}{1-2 t-t^{2}}\right)
$$

Moreover, if $D=\left[D_{n, k}\right]_{n, k \geq 0}$ is the Riordan matrix generated by the Delannoy numbers and $B=\left[\left(\begin{array}{l}n \\ k\end{array}\right)\right]_{n, k \geq 0}$ is the Riordan matrix generated by the binomial coefficients, then we have the decomposition $Q=D B$.

Proof. By series (5), we have at once Riordan matrix (15). Then, by identity (14), we immediately have the decomposition $Q=D B$.

Let $p(x)$ and $q(x)$ be two real polynomials with degree $n$ and $n+1$, with real distinct roots. Let $r_{1}<\cdots<r_{n}$ be the roots of $p(x)$ and let $s_{1}<\cdots<s_{n+1}$ be the roots of $q(x)$. The polynomials $p(x)$ and $q(x)$ strictly interlace if $s_{1}<r_{1}<s_{2}<r_{2}<\cdots<s_{n}<r_{n}<s_{n+1}$.

A standard polynomial is a real polynomial which is identically zero or with positive leading coefficient. A sequence $\left\{p_{n}(x)\right\}_{n \in \mathbb{N}}$ of standard polynomials is a Sturm sequence [16] when every $p_{n}(x)$ has degree $n$ and $p_{n-1}\left(x_{0}\right) p_{n+1}\left(x_{0}\right)<0$ whenever $p_{n}\left(x_{0}\right)=0$ and $n \geq 1$. These sequences can be characterized as follows: $\left\{p_{n}(x)\right\}_{n \in \mathbb{N}}$ is a Sturm sequence if and only if every polynomial $p_{n}(x)$ has degree $n$, has $n$ real distinct roots and strictly interlaces $p_{n+1}(x)$.

Theorem 19. The cube polynomials $q_{n}(x)$ form a Sturm sequence.

Proof. Let $q_{-1}(x)=0$ and

$$
f_{n}(x)=\left|\begin{array}{cc}
q_{n+1}(x) & q_{n}(x) \\
q_{n}(x) & q_{n-1}(x)
\end{array}\right|
$$

By recurrence (11), we have $f_{n+1}(x)=-(1+x) f_{n}(x)$ and consequently $f_{n}(x)=(-1-$ $x)^{n} f_{0}(x)$. Since $q_{0}(x)=1$ and $q_{1}(x)=2+x$, we have $f_{0}(x)=-1$ and then $f_{n}(x)=$ $-(-1-x)^{n}$, that is

$$
q_{n-1}(x) q_{n+1}(x)-q_{n}(x)^{2}=-(-1-x)^{n} .
$$


Hence, if $x_{0}$ is a root of $q_{n}(x)$, then we have $q_{n-1}\left(x_{0}\right) q_{n+1}\left(x_{0}\right)=-\left(-1-x_{0}\right)^{n}$. Since $q_{n}(x)=d_{n}(1+x)$ and $d_{n}(x)$ is a polynomial with positive coefficients $(n \geq 1)$, then $1+x_{0}$ is a root of $d_{n}(x)$ and $1+x_{0}<0$. So $q_{n-1}\left(x_{0}\right) q_{n+1}\left(x_{0}\right)<0$ for every $n \geq 1$. Since the polynomials $q_{n}(x)$ have degree $n$, they form a Sturm sequence.

REMARK 20. Similarly, also the Delannoy polynomials form a Sturm sequence.

A sequence $\left\{a_{0}, a_{1}, \ldots, a_{n}\right\}$ of (positive) real numbers is unimodal when there exists an index $k$ such that $a_{0} \leq a_{1} \leq \cdots \leq a_{k} \geq a_{k+1} \geq \cdots \geq a_{n}$, while it is log-concave when $a_{k+1} a_{k-1} \leq a_{k}^{2}$ for every $k \in\{1,2, \ldots, n-1\}$. A polynomial is unimodal (resp. log-concave) when the sequence of its coefficients is unimodal (resp. log-concave).

Theorem 21. The cube polynomials $q_{n}(x)$ are log-concave and unimodal.

Proof. A real polynomial all of whose roots are real and negative is log-concave [30, p. 137]. By Theorem 19, the polynomial $q_{n}(x)$ has $n$ real negative roots, and consequently is logconcave. Moreover, a log-concave sequence of positive numbers is unimodal [30, p. 137]. So, the polynomial $q_{n}(x)$ is also unimodal.

The cube numbers $q_{n}$, whose first few values are $1,3,11,39,139,495,1763,6279,22363$, 79647, 283667, 1010295, 3598219, form sequence A007482 in [28]. In particular, we have

Proposition 22. The cube numbers of the Pell graphs satisfy the recurrence

$$
q_{n+2}=3 q_{n+1}+2 q_{n}
$$

with the initial values $q_{0}=1$ and $q_{1}=3$. Moreover, they have generating series

$$
q(t)=\sum_{n \geq 0} q_{n} t^{n}=\frac{1}{1-3 t-2 t^{2}}
$$

and they can be expressed as

$$
q_{n}=\sum_{k=0}^{n} D_{n, k} 2^{k} .
$$

Proof. For $x=1$, recurrence (11), series (12) and identity (13) become recurrence (16), series (17) and identity (18), respectively.

REMARK 23. Clearly, recurrence (11) can also be obtained by a direct combinatorial argument. Indeed, if $Q$ is a subhypercube of $\Pi_{n+2}$, then we have the following possibilities. (i) $Q$ is contained in one of the three components of the canonical decomposition of $\Pi_{n+2}$. (ii) $Q=Q^{\prime} \oplus Q^{\prime \prime}$, where $Q^{\prime}$ is a hypercube contained in the first component and $Q^{\prime \prime}$ is the corresponding hypercube contained in the second component. $Q$ is completely determined by $Q^{\prime}$. (iii) $Q=Q^{\prime} \oplus Q^{\prime \prime}$, where $Q^{\prime}$ is a hypercube contained in the second component and $Q^{\prime \prime}$ is the corresponding hypercube contained in the third component. $Q$ is completely determined by $Q^{\prime \prime}$. Since there are no other possibilities, we have recurrence (16). 


\subsection{Cube indices}

The index $i(G)$ of a graph $G$ is the quotient between the number of edges and the number of vertices, i.e. $i(G)=|E(G)| /|V(G)|$. For the hypercube $Q_{n}$ we have $2^{n}$ vertices and $n 2^{n-1}$ edges, and index $i\left(Q_{n}\right)=n / 2$. This is true also for the Pell graphs. Indeed, by identity (9), we have $i\left(\Pi_{n}\right)=\ell_{n} / p_{n}=n / 2$. Hence $i\left(\Pi_{n}\right) \rightarrow+\infty$ as $n \rightarrow+\infty$. Such a result holds also for Fibonacci and Lucas cubes [23]. We define the cube index of order $k$ of a graph $G$ as

$$
i_{k}(G)=\frac{q_{k+1}(G)}{q_{k}(G)} .
$$

Since $i(G)=q_{1}(G) / q_{0}(G)$, we have $i_{0}(G)=i(G)$.

Theorem 24. For every fixed $k \in \mathbb{N}$, we have the asymptotic equivalences

$$
i_{k}\left(Q_{n}\right) \sim \frac{n}{2(k+1)} \quad \text { and } \quad i_{k}\left(\Pi_{n}\right) \sim \frac{n}{2(k+1)} \quad \text { for } n \rightarrow+\infty
$$

and, consequently, the limits

$$
\lim _{n \rightarrow+\infty} i_{k}\left(Q_{n}\right)=\lim _{n \rightarrow+\infty} i_{k}\left(\Pi_{n}\right)=+\infty .
$$

Proof. For the hypercubes we have $q_{k}\left(Q_{n}\right)=\left(\begin{array}{l}n \\ k\end{array}\right) 2^{n-k}$. So, we have

$$
i_{k}\left(Q_{n}\right)=\frac{q_{k+1}\left(Q_{n}\right)}{q_{k}\left(Q_{n}\right)}=\frac{n}{2(k+1)}\left(1-\frac{k}{n}\right) \sim \frac{n}{2(k+1)} \quad \text { for } n \rightarrow+\infty .
$$

For the Pell graphs, we will obtain a first-order asymptotic formula for $q_{n, k}=q_{k}\left(Q_{n}\right)$, with $k$ fixed, by using the following property [2, p. 252]. Given a complex number $\xi \neq 0$ and a complex function $f(t)$ analytic at the origin, if $f(t)=(1-t / \xi)^{-\lambda} \psi(t)$, where $\psi(t)$ is a series with radius of convergence $R>|\xi|$ and $\lambda \notin\{0,-1,-2, \ldots\}$, then we have

$$
\left[t^{n}\right] f(t) \sim \frac{\psi(\xi)}{\xi^{n}} \frac{n^{\lambda-1}}{\Gamma(\lambda)} \quad \text { for } n \rightarrow+\infty
$$

By series (5), we have

$$
Q_{k}(t)=\sum_{n \geq 0} q_{n, k} t^{n}=\frac{\left(t+t^{2}\right)^{k}}{\left(1-2 t-t^{2}\right)^{k+1}}=t^{k}(1+t)^{k}\left(1+\frac{t}{\alpha}\right)^{-k-1}\left(1+\frac{t}{\beta}\right)^{-k-1}
$$

where $\alpha=1+\sqrt{2}$ and $\beta=1-\sqrt{2}$. So, the dominant singularity is $\xi=-\beta, \lambda=k+1$ and

$$
\psi(t)=\left(t+t^{2}\right)^{k}(1+t / \alpha)^{-k-1} .
$$

Since $\psi(\xi)=\psi(-\beta)=\frac{2+\sqrt{2}}{2^{k+2}}=\frac{\alpha}{2^{k+1} \sqrt{2}}$ and $\alpha \beta=-1$, we have

$$
q_{n, k} \sim \frac{\psi(-\beta)}{(-\beta)^{n}} \frac{n^{k}}{\Gamma(k+1)}=\frac{1}{k !}\left(\frac{n}{2}\right)^{k} \frac{\alpha^{n+1}}{2 \sqrt{2}} \quad \text { for } n \rightarrow+\infty
$$

and consequently

$$
i_{k}\left(\Pi_{n}\right)=\frac{q_{n, k+1}}{q_{n, k}} \sim \frac{n}{2(k+1)} \quad \text { for } n \rightarrow+\infty .
$$

This is the second equivalence stated in (19). 


\subsection{Decompositions in hypercubes}

For the Pell numbers, we have the numerical identity

$$
p_{n}=\sum_{k=0}^{\lfloor n / 2\rfloor}\left(\begin{array}{c}
n-k \\
k
\end{array}\right) 2^{n-2 k} .
$$

In the next theorem, we give a combinatorial interpretation of this identity in terms of Pell graphs and hypercubes.

Theorem 25. The Pell graph $\Pi_{n}$ can be decomposed in $f_{n-1}$ hypercubes, i.e.

$$
\Pi_{n}=\bigoplus_{k=0}^{\lfloor n / 2\rfloor}\left(\begin{array}{c}
n-k \\
k
\end{array}\right) Q_{n-2 k} .
$$

Proof. Let $[n]=\{1,2, \ldots, n\}$, for any $n \in \mathbb{N}$. Given a Pell string $\alpha=a_{1} \cdots a_{n} \in \mathcal{P}_{n}$, consider the set $\mathcal{S}(\alpha)=\left\{i \in[n]: a_{i}=2\right\}$. For instance, if $\alpha=022122220122220$, then $\mathcal{S}(\alpha)=\{2,3,5,6,7,8,11,12,13,14\}$. We say that $\mathcal{S}(\alpha)$ is a Pell subset, i.e. a subset of $[n]$ where the consecutive elements form blocks of even length. Given a Pell subset $S \subseteq[n]$, let $\mathcal{P}_{n}(S)=\left\{\alpha \in \mathcal{P}_{n}: \mathcal{S}(\alpha)=S\right\}$. In the strings of $\mathcal{P}_{n}(S)$ the 2's are fixed and the variable letters are 0 and 1 . For instance, if $S=\{2,3,5,6,7,8\} \subseteq[10]$, the strings in $\mathcal{P}_{10}(S)$ have the form $\alpha=x_{1} 22 x_{2} 2222 x_{3} x_{4}$, where $x_{1}, x_{2}, x_{3}, x_{4} \in\{0,1\}$. So, the set $\mathcal{P}_{n}(S)$ induces a subgraph of $\Pi_{n}$ isomorphic to a hypercube $Q_{n-|S|}$. Clearly, if $S \neq T$, then $\mathcal{P}_{n}(S) \cap \mathcal{P}_{n}(T)=\varnothing$, that is the subhypercubes of $\Pi_{n}$ induced by $\mathcal{P}_{n}(S)$ and $\mathcal{P}_{n}(T)$ are vertex-disjoint.

Now, we have to count the Pell subset $S \subseteq[n]$ with $|S|=2 k$. If we represent $S$ by a binary string, then we have a binary string of length $n$ with $2 k 1$ 's where the maximal blocks of 1's have even length. Replacing all elementary factors 11 with 1, we have an arbitrary binary string of length $n-k$ with $k 1$ 's. So, we have $\left(\begin{array}{c}n-k \\ k\end{array}\right)$ Pell subsets $S \subseteq[n]$ with $|S|=2 k$, and, consequently, we have decomposition (21). The total number of the hypercubes appearing in such a decomposition is given by the Fibonacci number

$$
\sum_{k=0}^{\lfloor n / 2\rfloor}\left(\begin{array}{c}
n-k \\
k
\end{array}\right)=f_{n-1} .
$$

See Figure 4 for an example.

The quotient graph $\widetilde{\Pi}_{n}$ associated with the decomposition (21) is the simple graph where the vertices are the blocks (hypercubes) and two vertices are adjacent when there is at least one edge connecting the corresponding blocks.

Theorem 26. The quotient graph $\widetilde{\Pi}_{n}$ is isomorphic to the Fibonacci cube $\Gamma_{n-1}(n \geq 1)$.

Proof. The vertices of $\widetilde{\Pi}_{n}$ correspond to the sets $\mathcal{P}_{n}(S)$, where $S \subseteq[n]$ is a Pell subset. Since the maximal blocks of consecutive elements are of even length, to give such a subset is equivalent to give a sparse subset (or scattered subset) of $[n]$, i.e. a subset of $[n]$ with no 


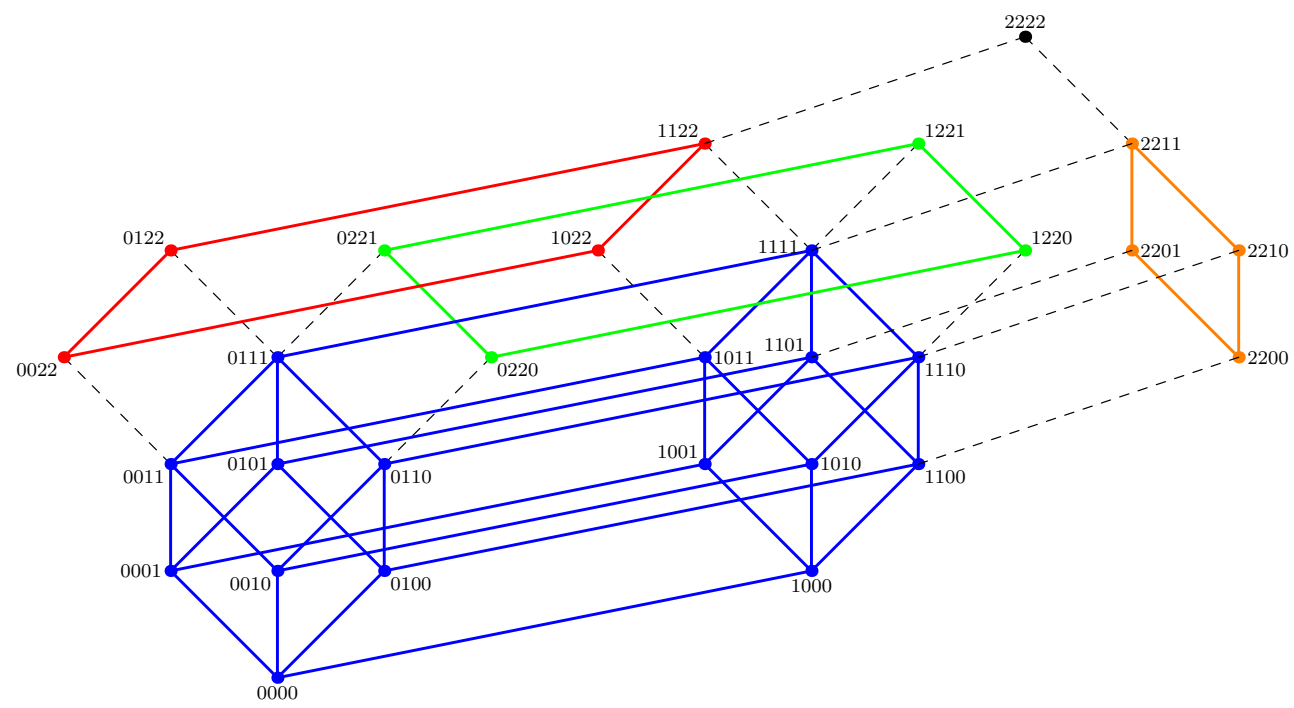

Figure 4: Decomposition in hypercubes of $\Pi_{4}: \Pi_{4}=Q_{4} \oplus Q_{2} \oplus Q_{2} \oplus Q_{2} \oplus Q_{0}$

consecutive elements. For instance, the subset $S=\{2,3,5,6,7,8,11,12,13,14\} \subseteq[10]$ is equivalent to the sparse subset $S^{\prime}=\{2,5,7,11,13\} \subseteq[10]$. So, $\mathcal{P}_{n}(S)$ and $\mathcal{P}_{n}(T)$ are two adjacent vertices in the quotient graph exactly when the corresponding sparse subsets $S^{\prime}$ and $T^{\prime}$ differ only for one element, i.e. $S^{\prime}=T^{\prime} \cup\{*\}$ or $T^{\prime}=S^{\prime} \cup\{*\}$. This implies that the quotient graph is isomorphic to the graph where the vertices are the $f_{n-1}$ sparse subsets of $[n]$ and two vertices are adjacent whenever they differ for a single element. This last graph is isomorphic to the Fibonacci cube $\Gamma_{n-1}$.

\section{Distribution of the degrees}

The distribution of the degrees in Fibonacci and Lucas cubes has been studied in [21]. Here, we study the distribution of the degrees in a Pell graph. By the definition of adjacency in a Pell graph, we have that the degree of a vertex $\alpha \in \Pi_{n}$ is

$$
d(\alpha)=\omega_{0}(\alpha)+\omega_{1}(\alpha)+\omega_{11}(\alpha)+\frac{1}{2} \omega_{2}(\alpha)
$$

where $\omega_{\xi}(\alpha)$ is the number of occurrences of the factor $\xi$ in $\alpha$. For instance, $d(01222200111)=$ $3+4+2+2=11$.

Proposition 27. The maximum and the minimum degree of $\Pi_{n}$ are $\Delta\left(\Pi_{n}\right)=2 n-1$ (for $n \geq 1)$ and $\delta\left(\Pi_{n}\right)=\left\lceil\frac{n}{2}\right\rceil$, respectively. The expected degree in a random vertex of $\Pi_{n}$ is $n$.

Proof. The maximum degree is reached when we have the maximum number of 1 's, i.e. with the string $1^{n}=11 \cdots 11$. Every 1 can be changed to 0 and every factor 11 can be changed to 22 . So $\Delta=n+n-1=2 n-1$. The minimum degree is reached when we have the maximum number of 2 's. If $n=2 k$, then we have the string $2^{n}=22 \cdots 22$. In this case, 
every factor 22 can be changed to 11 and $\delta=k$. If $n=2 k+1$, then we have, for instance, the string $2^{n}=22 \cdots 220$. In this case, every factor 22 can be changed to 11 and 0 can be changed to 1 . So $\delta=k+1$. In conclusion, $\delta=\lceil n / 2\rceil$. Finally, by identity (9), the medium degree of a vertex of $\Pi_{n}$ is $\frac{1}{\left|\Pi_{n}\right|} \sum_{v \in \Pi_{n}} d(v)=\frac{2 \ell_{n}}{p_{n}}=n$.

The chromatic index $\chi^{\prime}(G)$ of a graph $G$ is the minimum number of colors for which there exists a proper edge coloring [1].

Proposition 28. The chromatic index of the Pell graph $\Pi_{n}$ is $\chi^{\prime}\left(\Pi_{n}\right)=2 n-1$ (for $n \geq 1$ ).

Proof. By König's Coloring Theorem [1], the chromatic index $\chi^{\prime}(G)$ of a bipartite graph $G$ is equal to the maximum degree $\Delta(G)$ of $G$. Since $\Pi_{n}$ is bipartite (Theorem 1) and $\Delta\left(\Pi_{n}\right)=2 n-1$ (Theorem 27 ), the claim follows.

Let $\Delta_{n, k}$ be the number of all vertices of $\Pi_{n}$ having degree $k$, and let $\Delta_{n}(x)=\sum_{k \geq 0} \Delta_{n, k} x^{k}$ be the associated polynomials, with generating series

$$
\Delta(x, t)=\sum_{n \geq 0} \Delta_{n}(x) t^{n}=\sum_{n, k \geq 0} \Delta_{n, k} x^{k} t^{n} .
$$

Theorem 29. The polynomials $\Delta_{n}(x)$ have generating series

$$
\Delta(x, t)=\frac{1+\left(x-x^{2}\right) t}{1-\left(x+x^{2}\right) t-\left(x+x^{2}-x^{3}\right) t^{2}-\left(x^{2}-x^{3}\right) t^{3}} .
$$

and satisfy the recurrence

$$
\Delta_{n+3}(x)=\left(x+x^{2}\right) \Delta_{n+2}(x)+\left(x+x^{2}-x^{3}\right) \Delta_{n+1}(x)+\left(x^{2}-x^{3}\right) \Delta_{n}(x)
$$

with the initial values $\Delta_{0}(x)=1, \Delta_{1}(x)=2 x$ and $\Delta_{2}(x)=x+3 x^{2}+x^{3}$. Moreover, we have the recurrence

$$
\Delta_{n+3, k+3}=\Delta_{n+2, k+2}+\Delta_{n+2, k+1}+\Delta_{n+1, k+2}+\Delta_{n+1, k+1}-\Delta_{n+1, k}+\Delta_{n, k+1}-\Delta_{n, k}
$$

with the initial values $\Delta_{n, 0}=\delta_{n, 0}, \Delta_{n, 1}=2 \delta_{n, 1}+\delta_{n, 2}, \Delta_{n, 2}=3 \delta_{n, 2}+4 \delta_{n, 3}+\delta_{n, 4}, \Delta_{0, k}=\delta_{k, 0}$, $\Delta_{1, k}=2 \delta_{k, 1}$ and $\Delta_{2, k}=\delta_{k, 1}+3 \delta_{k, 2}+\delta_{k, 3}$.

Proof. Let $\mathcal{P}_{1}$ be the set of all Pell strings starting with 1 , and let $\Delta_{1}(x, t)$ be the associated generating series. Then, we have the system

$$
\left\{\begin{array}{l}
\mathcal{P}=\varepsilon+0 \mathcal{P}+\mathcal{P}_{1}+22 \mathcal{P} \\
\mathcal{P}_{1}=1\left(\varepsilon+0 \mathcal{P}+\mathcal{P}_{1}+22 \mathcal{P}\right)
\end{array}\right.
$$

from which, by using the definition of degree in a Pell graph, we obtain the system

$$
\left\{\begin{array}{l}
\Delta(x, t)=1+x t \Delta(x, t)+\Delta_{1}(x, t)+x t^{2} \Delta(x, t) \\
\Delta_{1}(x, t)=x t\left(1+x t \Delta(x, t)+x \Delta_{1}(x, t)+x t^{2} \Delta(x, t)\right) .
\end{array}\right.
$$

By solving this system, we obtain series (23), and the form of this rational series implies at once recurrences (24) and (25). 
The first values of the numbers $\Delta_{n, k}$ appear in the matrix

$$
F=\left[\begin{array}{ccccccccccccccccc}
1 & & & & & & & & & & & & & & \\
0 & 2 & & & & & & & & & & & & & \\
0 & 1 & 3 & 1 & & & & & & & & & & & \\
0 & 0 & 4 & 5 & 2 & 1 & & & & & & & & & \\
0 & 0 & 1 & 10 & 10 & 5 & 2 & 1 & & & & & & & \\
0 & 0 & 0 & 6 & 22 & 21 & 12 & 6 & 2 & 1 & & & & & \\
0 & 0 & 0 & 1 & 21 & 48 & 45 & 29 & 15 & 7 & 2 & 1 & & & \\
0 & 0 & 0 & 0 & 8 & 59 & 106 & 99 & 68 & 39 & 18 & 8 & 2 & 1 & \\
0 & 0 & 0 & 0 & 1 & 36 & 151 & 236 & 222 & 160 & 96 & 50 & 21 & 9 & 2 & 1 \\
\cdots & & & & & & & & & & & & & &
\end{array}\right]
$$

Let $\Delta_{n}=\Delta_{n, n}$ be the number of vertices of $\Pi_{n}$ with degree $n$ (i.e. with medium degree). The first few values are: $1,2,3,5,10,21,45,99,222,503,1148,2636,6082$. Using Cauchy's integral formula to calculate the diagonal of a bivariate series [10] and employing standard algebraic techniques on formal series, it is possible to obtain the following results.

Theorem 30. The numbers $\Delta_{n}=\Delta_{n, n}$ have generating series

$$
\Delta(t)=\sum_{n \geq 0} \Delta_{n} t^{n}=\frac{1-2 t-2 t^{3}-t^{4}+(1+t) \sqrt{1-2 t-t^{2}-t^{4}+2 t^{5}+t^{6}}}{2\left(1-3 t+2 t^{2}-2 t^{3}+t^{4}+t^{5}\right)}
$$

and satisfy the recurrence

$$
\begin{aligned}
& (n+7) \Delta_{n+7}-3(n+7) \Delta_{n+6}+(n+13) \Delta_{n+5}+(n-5) \Delta_{n+4}+ \\
& \quad-(n-3) \Delta_{n+3}+(3 n+7) \Delta_{n+2}-(n+3) \Delta_{n+1}-(n+1) \Delta_{n}=0
\end{aligned}
$$

with the initial values $\Delta_{0}=1, \Delta_{1}=2, \Delta_{2}=3, \Delta_{3}=5, \Delta_{4}=10, \Delta_{5}=21$ and $\Delta_{6}=45$.

[1] A. S. Asratian, T. M. J. Denley, R. Häggkvist, Bipartite Graphs and Their Applications, Cambridge Tracts in Mathematics, 131, Cambridge University Press, Cambridge, 1998.

[2] F. Bergeron, G. Labelle, P. Leroux, Combinatorial Species and Tree-like Structures, Encyclopedia of Mathematics and Its Applications 67, Cambridge University Press, Cambridge, 1998.

[3] J. A. Bondy, U. S. R. Murty, Graph Theory, Springer, New York, 2008.

[4] B. Brešar, S. Klavžar, R. Škrekovski, The cube polynomial and its derivatives: the case of median graphs, Electron. J. Comb. 10 (2003).

[5] F. Buckley, F. Harary, Distance in Graphs, Addison-Wesley, Redwood City, 1990.

[6] F. Buckley, F. Harary, Unsolved problems on distance in graphs, Electron. Notes Discrete Math. 11, Elsevier, Amsterdam, 2002.

[7] L. Comtet, Advanced Combinatorics, Reidel, Dordrecht-Holland, Boston, 1974.

[8] P. Flajolet, R. Sedgewick, Analytic Combinatorics, Cambridge University Press, Cambridge, 2009.

[9] G. Hahn, C. Tardif, Graph homomorphisms: structure and symmetry, 107-166, in: G. Hahn, G. Sabidussi (Eds.), Graph Symmetry, Kluver, 1997.

[10] M. L. J. Hautus, D. A. Klarner, The diagonal of a double power series, Duke Math. J. 38 (1971) 229-235.

[11] W.-J. Hsu, Fibonacci cubes - A new computer architecture for parallel processing, Technical Report CPS-90-04, Michigan State University 1990. 
[12] W.-J. Hsu, Fibonacci cubes - A new interconnection topology, IEEE Trans. on Parallel and Distributed Systems 4 (1993) 3-12.

[13] W. Imrich, S. Klavžar, Product Graphs. Structure and Recognition, Wiley-Interscience Series in Discrete Mathematics and Optimization. Wiley-Interscience, New York, 2000.

[14] A. Ilićc, S. Klavžar, Y. Rho, Generalized Fibonacci cubes, Discrete Math. 312 (2012) 2-11.

[15] A. Ilićc, S. Klavžar, Y. Rho, Generalized Lucas cubes, Appl. Anal. Discrete Math. 6 (2012) 82-94.

[16] L. L. Liu, Y. Wang, A unified approach to polynomial sequences with only real zeros, Adv. in Appl. Math. 38 (2007) 542-560.

[17] J. Liu, W.-J. Hsu, M. J. Chung, Generalized Fibonacci cubes are mostly Hamiltonian, J. Graph Theory 18 (1994) 817-829.

[18] S. Klavžar, On median nature and enumerative properties of Fibonacci-like cubes, Discrete Math. 299 (2005) 145-153.

[19] S. Klavžar, Structure of Fibonacci cubes: a survey, J. Comb. Optim. 25 (2013) 505-522.

[20] S. Klavžar, M. Mollard, Cube polynomial of Fibonacci and Lucas cubes, Acta Appl. Math. 117 (2012) 93-105.

[21] S. Klavžar, M. Mollard, M. Petkovšek, The degree sequence of Fibonacci and Lucas cubes, Discrete Math. 311 (2011) 1310-1322.

[22] M. Mulder, The structure of median graphs, Discrete Math. 24 (1978) 197-204.

[23] E. Munarini, C. Perelli Cippo, N. Zagaglia Salvi, On the Lucas cubes, Fibonacci Quart. 39 (2001) $12-21$.

[24] E. Munarini, N. Zagaglia Salvi, Structural and enumerative properties of the Fibonacci cubes, Discrete Math. 255 (2002) 317-324.

[25] E. Munarini, N. Zagaglia Salvi, On Fibonacci-like cubes, Rend. Sem. Mat. Messina Ser. II 9 (2003) $185-199$.

[26] H. Quian, J. Wu, Enhanced Fibonacci cubes, Comput. J. 39 (1996) 331-345.

[27] L. W. Shapiro, S. Getu, W. J. Woan, L. C. Woodson, The Riordan group, Discrete Appl. Math. 34 (1991) 229-239.

[28] N. J. A. Sloane, On-Line Encyclopedia of Integer Sequences, http://oeis.org/.

[29] C. Whitehead, N. Zagaglia Salvi, Extended Lucas cubes, Util. Math. 71 (2006) 13-21.

[30] H. S. Wilf, Generatingfunctionology, Academic Press, Boston, 1990.

[31] J. Wu, Extended Fibonacci cubes, IEEE Trans. Parallel Distributed Systems 8 (12) (1997) 3-9. 